\title{
Active site cleft mutants of Os9BGlu31 transglucosidase modify acceptor substrate specificity and allow production of multiple kaempferol glycosides
}

Juthamath Komvongsa $^{a}$, Sukanya Luang ${ }^{a}$, Joaquim V. Marques ${ }^{b}$, Kannika Phasai ${ }^{a}$, Laurence B. Davin $^{\mathrm{b}}$, Norman G. Lewis ${ }^{\mathrm{b}}$, and James R. Ketudat Cairns ${ }^{\mathrm{a}, \mathrm{c}^{*}}$

${ }^{\mathrm{a}}$ School of Biochemistry, Institute of Science, and Center for Biomolecular Structure, Function and Application, Suranaree University of Technology, Nakhon Ratchasima 30000, Thailand.

${ }^{\mathrm{b}}$ Institute of Biological Chemistry, Washington State University, Pullman, WA 99164, USA.

${ }^{c}$ Laboratory of Biochemistry, Chulabhorn Research Institute, Bangkok, Thailand

* Corresponding Author; email: cairns@sut.ac.th, Tel. +66 44 224304; Fax +66 44224185 


\begin{abstract}
Background: Rice Os9BGlu31 is a transglucosidase that can transfer glucose to phenolic acids, flavonoids, and phytohormones. Os9BGlu31 displays a broad specificity with phenolic 1-O- $\beta$-Dglucose esters acting as better glucose donors than glucosides, whereas the free phenolic acids of these esters are also excellent acceptor substrates. Methods: Based on homology modeling of this enzyme, we made single point mutations of residues surrounding the acceptor binding region of the Os9BGlu31 active site. Products of the wild type and mutant enzymes in transglycosylation of phenolic acceptors from 4-nitrophenyl $\beta$-D-glucopyranoside donor were identified and measured by UPLC and negative ion electrospray ionization tandem mass spectrometry (LCMSMS). Results: The most active variant produced was W243N, while I172T and L183Q mutations decreased the activity, and other mutations at W243 (A, D, M, N, F and Y) had variable effects, depending on the acceptor substrate. The Os9BGlu31 W243N mutant activity was higher than that of wild type on phenolic acids and kaempferol, a flavonol containing 4 hydroxyl groups, and the wild type Os9BGlu31 produced only a single product from each of these acceptors in significant amounts, while W243 variants produced multiple glucoconjugates. Fragmentation analysis provisionally identified the kaempferol transglycosylation products as 3-O,7-O, and 3,7-O, 4',7-O, and 3,4'-O kaempferol bis- $O$ glucosides. The Os9BGlu31 W243 mutants were also better able to use kaempferol 3-Oglucoside as a donor substrate. Significance: The W243 residue was found to be critical to the substrate and product specificity of Os9BGlu31 transglucosidase and mutation of this residue allows production of a range of glucoconjugates.
\end{abstract}

Keywords: rice (Oryza sativa L.), transglucosidase, site-directed mutagenesis, Glycoside hydrolase family GH1, glycosylation, electrospray ionization mass spectrometry 


\section{Introduction}

Glycosylation is an important mechanism involved in controlling the bioactivity of many phytohormones and plant metabolites, including detoxification. The formation and cleavage of glycosidic linkages in such conjugates can be mediated by glycosyltransferases (GTs), glycoside hydrolases (GHs), or transglycosidases (TGs) [1-3]. Glycosidic bonds can be formed by both glycosyltransferases, which use nucleotide or phospholipid sugars as donor substrates, and transglycosidases, which transfer sugars from other donor substrates. Transfer of glycosyl moieties to water (in hydrolysis) or to form glycoconjugates (in transglycosylation) can occur with retention or inversion of the configuration of the anomeric carbon in the glycosidic bond, but the TGs described to date are primarily retaining enzymes [4-5]. There are a large number of retaining GHs that catalyze both hydrolysis and transglycosylations, but not much is known regarding what determines the ratio between the two types of reactions [6].

Glycosynthases, which transfer sugars from anomeric fluoroglucosides, are typically produced by mutation of a retaining GH catalytic nucleophile to a small non-nucleophilic group [7,8], while thioglycoligases, which transfer from an activated donor to a thiol (or other nucleophilic) acceptor, are typically made by mutation of the catalytic acid/base [9]. These mutational approaches modify the mechanism to disfavor hydrolysis and therefore improve transglycosylation yields. Other groups have worked to convert GH to TG [6] or enhance the rate and substrate specificity of natural transglycosidases, such as amylosucrases and cyclodextrinases, by directed evolution [10]. In GH family GH16, a surface loop thought to affect acceptor substrate binding was identified to help differentiate between a xyloglucan endohydrolase and xyloglucan endotransferase (XET, a TG) [11], but the critical determinant of transglycosylation activity is generally unclear.

Although GH family GH1 enzymes have long been known to catalyze transglycosylation in addition to hydrolysis, natural TGs belonging to GH1 have recently been described, including transgalactosidases and transglucosidases [5, 12]. Galactolipid-galactolipid galactosyl transferase, a chloroplast outer membrane enzyme that remodels glycolipids, was recently found to correspond to the GH1 protein SFR2 [12]. GH1 enzymes were also found to be acyl glucose dependent anthocyanin glucosyl transferases (AAGTs, which in fact are TGs), 
responsible for transferring a second glucosyl moiety to anthocyanidin 3-O-glucoside in certain flowers [11]. AAGTs were subsequently demonstrated to synergistically participate with acylglucose dependent acyl transferases to build up large anthocyanin complexes by a series of sugar and acyl transfers to the anthocyanin backbone [13]. The Arabidopsis GH1 enzyme AtBGLU10 was shown to play a similar anthocyanin TG role [14].

Rice Os9BGlu31 is a vacuolar TG belonging to GH1 that acts to transfer glucosyl moieties between phenolic acids, phytohormones, and flavonoids [15]. It shows highest activity with feruloyl glucose and similar phenolic acid esters as donors, whereas the free phenolic acids of these esters are also excellent acceptor substrates. When no acceptor is present, 4-nitrophenyl$\beta$-D-glucoside (4NPGlc) hydrolysis by a thioredoxin-Os9BGlu31 fusion protein is approximately $10 \%$ of its transglycosylation activity in the presence of $0.25 \mathrm{mM}$ ferulic acid, but in the presence of such acceptors insignificant amounts of glucose are released. This suggests that Os9BGlu31 may help modulate the free phenolic acid and phenolic acid conjugate levels in plants, which may play a role in plant growth and cell wall synthesis [15]. Although Os9BGlu31 does not transfer glucose to cyanidin 3-O-glucoside, the preferred acceptor substrate of acyl glucosedependent anthocyanin transglucosidases from carnation and delphinium [5], it is able to use another flavonoid, apigenin 7-O-glucoside, as a glucosyl donor and apigenin as an acceptor [15]. Rice Os9BGlu31 displays an unusual lack of inhibition by mechanism-based inhibitors of GH1 $\beta$-glucosidases. However, mutagenesis showed that the E169 and E387 residues, corresponding to the conserved catalytic acid-base and nucleophile in GH1 glycosidases, are important for Os9BGlu31 catalysis, supporting the idea that it uses a double displacement retaining mechanism similar to GH1 $\beta$-glucosidases. The residue preceding the catalytic nucleophile is typically a threonine in plant GH1 $\beta$-glucosidases, but is a histidine (H368) in Os9BGlu31 transglucosidase. However mutating $\mathrm{H} 368$ to $\mathrm{T}$ had no effect on the ratio of transglycosylation to hydrolysis.

Flavonoids are a widely distributed group of natural products found in plants. They are classified according to their chemical structure. Flavones and flavonols are classified as anthoxanthins (from the Greek word "xanthos", meaning yellow) and have very similar core structures, but differences in the number and position of hydroxyl and methoxyl groups. They have potential health benefits arising from antioxidant, radical scavenger, anti-inflammatory, and 
antiviral activities [16-17]. Kaempferol is a flavonol with four hydroxyl groups at the 3, 5, 7, and $4^{\prime}$ positions, and it has been found to be glucosylated at the 3, 7, and 4' positions in different plant species [18]. Both flavonoid glycoside and aglycone structures have been widely characterized [19-21]. Electrospray ionization tandem mass spectrometry (ESI-MS/MS) has been used in the structural characterization of flavonoid glycosides (including their glycosylation patterns) and aglycones [22-25]. One consistent phenomenon described is the relationship between the formation of deprotonated kaempferol mono- $O$-glucoside and fragmentation of the radical aglycone ions in ESI-MS/MS and the nature and position of glycosyl substitution [26].

In this study, we have characterized the effects of mutations of residues surrounding the aglycone/acceptor binding site of Os9BGlu31 on glucosyl transfer to different acceptors. The product regio-specificity of variants mutated at W243 was also investigated in experiments with kaempferol as a model by monitoring the production of mono- and di-glucosides, which were identified from their mass spectra and differential fragmentation patterns.

\section{Materials and methods}

\subsection{Site-directed mutagenesis of Os $9 B G l u 31$}

Mutations were produced according to the instructions of the QuikChange XL II sitedirected mutagenesis kit (Agilent, USA). The primers were designed based on the homology model of Os9BGlu31 (Figure 1). The homology model was generated with the rice Os3BGlu6 crystal structure (PDB ID: 3GNO) [27] as template in the Modeller 9 program [28]. Five models were generated and the one with the lowest DOPE score was used for comparison with Os3BGlu6. The model was superimposed with the covalent 2-deoxy-2-fluoroglucoside complex of Os3BGlu6 (PDB code: 3GNR) and visualized with PyMol (Schrödinger LLC, USA) to identify residues near the acceptor binding site. The pET32a/DEST/Os9BGlu31 vector was used as template to generate single point mutation of I172T, L183Q, L241D, and W243 to N, A, D, F, M, and Y. The primers used are given in Table S1 (Appendix A). The reaction was thermocycled as follows: $95^{\circ} \mathrm{C} 1 \mathrm{~min}$, then 18 cycles at $95^{\circ} \mathrm{C} 50 \mathrm{~s} ; 60^{\circ} \mathrm{C} 50 \mathrm{~s}$ and $68^{\circ} \mathrm{C} 7 \mathrm{~min}$, followed by a final extension at $68^{\circ} \mathrm{C} 7 \mathrm{~min}$. The templates were digested by Dpn I for $3 \mathrm{~h}$ and the resulting 
mutant plasmids were transformed into XL10-Gold Ultracompetent cells. Plasmids were prepared by standard methods and the full inserts sequenced to verify that only the intended mutations were present.

\subsection{Protein expression and purification}

The Os9BGlu31 proteins were expressed from the pET32a/DEST/Os9BGlu31 in Escherichia coli strain Origami ${ }^{\mathrm{TM}} \mathrm{B}(\mathrm{DE} 3)$ (Novogen, USA) and extracted from the cells as previously described $[15,29]$. The Os9BGlu31 protein was purified in 3 steps of IMAC, proteolytic cleavage of the tag and IMAC. First, crude protein was mixed with TALON Metal Affinity Resin (Clontech, USA) in equilibration buffer (150 mM NaCl, $20 \mathrm{mM}$ Tris- $\mathrm{HCl}, \mathrm{pH}$ 8.0) on ice for $30 \mathrm{~min}$. The resin with crude protein was loaded into a column and washed with 5 column volumes (CV) each of 5, 10 and $20 \mathrm{mM}$ imidazole in equilibration buffer. Os9BGlu31 fusion protein was eluted with $250 \mathrm{mM}$ imidazole in equilibration buffer. The fractions with activity were pooled, and imidazole was removed with Centricon $10 \mathrm{kDa}$ MWCO centrifugal filter units- (Millipore, USA) with 5 exchanges of 5 volumes equilibration buffer. The protein was digested with enterokinase light chain (New England BioLabs, USA) at $23^{\circ} \mathrm{C}$ for $16 \mathrm{~h}$. Finally, Os9BGlu31 protein was loaded into a TALON Metal Affinity Resin column, and the unbound Os9BGlu31 protein was collected and concentrated with Centricon $10 \mathrm{kDa}$ MWCO centrifugal filter units, as described above.

\subsection{Relative activities of Os9BGlu31 wild type and mutants}

The activities with various glucose acceptor substrates were assayed with $0.25 \mathrm{mM}$ acceptor, 2.5\% dimethylsulfoxide (DMSO, as solvent for the acceptors), $5 \mathrm{mM}$ 4-nitrophenyl $\beta$ D-glucopyranoside (4NPGlc) as glucose donor, and $0.25 \mu \mathrm{g}$ of Os9BGlu31 wild type and W243A, D, M, F, and Y mutants, $0.1 \mu \mathrm{g}$ of Os9BGlu31 W243N mutant, $1.25 \mu \mathrm{g}$ of Os9BGlu31 L183Q mutant or 5 $\mu \mathrm{g}$ of I172T mutant, variant in $50 \mathrm{mM}$ citrate buffer, $\mathrm{pH} 4.5$. For hydrolysis reactions, water replaced the acceptor and DMSO. The reactions were incubated at $30^{\circ} \mathrm{C}$ against reaction time, and then stopped by adding formic acid to $1 \%$. Time courses were done with all acceptors for the W243N mutant to establish the amount of product that could be produced with a linear response, corresponding to $\mathrm{v}_{0}$. Subsequently, reactions were run for $15 \mathrm{~min}$, at which time the product concentrations fell within the range that gave a linear response. The reactions were filtered with $0.22 \mu \mathrm{m}$ filters and separated through a 
ZORBAX SB-C18 (1.8 $\mu \mathrm{m}, 2.1 \times 150 \mathrm{~mm})$ column (Agilent) on an Agilent 1290 UPLC. The mobile phase consisted of $0.2 \%$ formic acid in water (solvent $A$ ) and $0.2 \%$ formic acid in acetonitrile (solvent B). The compounds were eluted with a linear gradient from 5 to $50 \%$ B (v/v) in $13.0 \mathrm{~min}, 50$ to $70 \% \mathrm{~B}(\mathrm{v} / \mathrm{v})$ in $1.00 \mathrm{~min}$, and 70 to $100 \% \mathrm{~B}(\mathrm{v} / \mathrm{v})$ in $2.00 \mathrm{~min}$ with flow rate of $0.2 \mathrm{~mL} / \mathrm{min}$. The diode array detector (DAD) was set to scan the range from 190-500 $\mathrm{nm}$ and the results were monitored and quantified at $360 \mathrm{~nm}$.

The protein absorbance was measured with a Nanodrop 2000c spectrophotometer (Thermo Scientific) at $280 \mathrm{~nm}$ and the protein concentration calculated from this absorbance with the extinction coefficient for each specific variant. The extinction coefficients were 1.72 for wild type, I172T and L183Q, 1.65 for W243Y, and 1.63 for other W243 mutants.

\subsection{Evaluation of transglucosylation of kaempferol with 4NPGlc and kaempferol 3-O-glucoside}

The products of transglycosylation by Os9BGlu31 and its W243 mutants were determined in reactions with $0.25 \mathrm{mM}$ kaempferol as glucose acceptor, 4NPGlc as donor, and 5 $\mu \mathrm{g}$ of wild type or mutant Os9BGlu31 in $50 \mathrm{mM}$ citrate buffer, $\mathrm{pH} 4.5$. In another set of reactions, $0.25 \mathrm{mM}$ kaempferol 3-O-glucoside, which was kindly provided by Prof. Dr. Toscahi Amahura, Matsuyama University, Japan, was used as the sole substrate of the enzyme. The products were quantified by ACQUITY UPLC system (Waters, USA) equipped with a DAD detector and a WATERS Xevo G2 QToF mass spectrometer in the negative ion mode, with a capillary voltage of $2 \mathrm{kV}$, a source temperature of $100^{\circ} \mathrm{C}$ and desolvation temperature of $200^{\circ} \mathrm{C}$. Desolvation gas flow was set as $600 \mathrm{~L} / \mathrm{h}$ with the lock spray infusion flow rate at $20 \mu \mathrm{L} / \mathrm{min}$ and lock spray capillary voltage at $2.50 \mathrm{kV}$. The collected data were analyzed with MassLynx Software.

\section{Results and discussion}

\subsection{Effect of mutations of W243, L183, and 1172 on Os9BGlu31 activity}


We hypothesized that the high transglycosylation versus hydrolysis activity of Os9BGlu31 transglucosidase might be due to poor binding of water relative to other acceptor substrates. To test this hypothesis, we attempted to increase hydrolysis at the expense of transglycosylation by changing active site cleft residues to more polar residues, in order to improve binding of water as an acceptor substrate. Comparison of the active site of a homology model of Os9BGlu31 with the X-ray crystal structure (PDB ID: 3GNR) of the 2-deoxy-2fluoroglucoside complex of Os3BGlu6, a GH1 $\beta$-glucosidase with no significant transglycosylation activity [27], identified several residues differing around the acceptor substrate binding site, as shown in Fig. 1. In the Os3BGlu6 structure, a water molecule (Wat642) is positioned $2.7 \AA$ from the catalytic acid/base and $3.6 \AA$ from the anomeric carbon, directly across from the bond to the catalytic nucleophile in this covalent intermediate complex. Three residues that were within $5.5 \AA$ of this water in the superimposed Os9BGlu31 model that were significantly less polar than the corresponding residues in Os3BGlu6 were I172, L183, and L241.To increase the polarity near the putative water-binding site, these were mutated to the corresponding residues in Os3BGlu6 (I172T, L183Q and L241D). We also mutated W243 to the $\mathrm{N}$ found in Os3BGlu7 $\beta$-glucosidase (W243N) in order to make the acceptor-binding site more hydrophilic. Although this residue side-chain is nearly $9 \AA$ from the putative acceptor water molecule in the superposition, it has been shown to have significant effects on the substrate specificities of Os3BGlu6, Os3BGlu7 and Os4BGlu12 $\beta$-glucosidases [30, 31].

The quantification of 4NP released from 4NPGlc in the presence of different acceptors by UPLC allowed the relative activities of these mutants for transglucosylation of various aromatic acceptors to be determined. The activities of the mutant enzymes Os9BGlu31 I172T and L183Q were lower and W243N higher than wild type (Fig. 2A), while the L241D mutant gave little soluble protein, so that it could not be characterized. It was noted that the I172T and L183Q mutants appeared to be less stable than the wild type and W243N enzymes, which may have contributed to their low activities.

The wild type Os9BGlu31 (Fig. 2B) and each of the initial mutated variants, I172T, L183Q and W243N (Figs. 2C-E) had highest activity with 4-hydroxybenzoic acid (4HBA) among the acceptor substrates tested. Compared to wild type Os9BGlu31 (Fig. 2B), only the 
W243N mutant (Fig. 2E) had a higher rate of hydrolysis (39\%) relative to transglycosylation of 4HBA (30\%, DMSO was not included in the hydrolysis reactions and the enzyme with the fusion tag removed may have been more sensitive to solvent effects, so hydrolysis rates appeared relatively high in the current comparison). We had hypothesized that mutant enzymes should have more hydrolysis activity than the wild type recombinant enzyme, due to the conversion of the residues to more hydrophilic amino acids, but all mutants still maintained higher rates of transglycosylation than hydrolysis. Since the binding of water or other acceptors in the active site involves complex interactions between the active site amino acid residues and the water network $[32,33]$, it may be that increasing the hydrophilicity of one nearby residue is not sufficient to convert a transglucosidase to a glucosidase. However, the mutant enzymes showed differences in acceptor specificity, as shown by the relative activities in Figs. 2B-E. For instance, I172T showed a high relative preference for 1-naphthol, while L183Q had a high relative preference for naphthalene acetic acid (NAA) compared to the other acceptors.

\subsection{Substrate specificity of Os9BGlu31 W243 mutants}

Both the wild type Os9BGlu31 and the W243N mutant transfer the glucosyl moiety from a donor substrate to an acceptor, but the Os9BGlu31 W243N enzyme has higher velocity and is more likely to transfer glucose to water compared to wild type Os9BGlu31. We have substituted the W243 position with alanine (small), methionine (hydrophobic, nonaromatic), phenylalanine (aromatic), tyrosine (aromatic hydroxyl), and aspartate (acidic) residues. Only minor effects on the optimum $\mathrm{pH}$ were observed, as shown in Fig. S1 (Appendix A). Moreover, the W243 mutant enzymes show multiple products in reactions with phenolic acceptors that have both carboxyl and hydroxyl groups that can act as acceptors, such as ferulic, 4-hydroxybenzoic, 4-coumaric, caffeic, sinapic, and vanillic acids (Fig. 3 and Appendix A Fig. S2). The W243N mutant could produce, for example, two products for each of these substrates, while the W243A mutant could only form detectable amounts of the products not observed with wild type Os9BGlu31 for ferulic and caffeic acids, as seen for ferulic acid in Fig. 3. Other mutants were intermediate in their abilities to produce the two products. These products were found to have the same $\mathrm{m} / \mathrm{z}$ but different retention times. Since these acceptors have only one hydroxyl (two for caffeic acid) and one carboxylic group to which to add glucose, the extra products not detected in the reactions 
with wild type Os9BGlu31 were surmised to be glucoside products, as most of these eluted from the $\mathrm{C} 18$ column before the glucose ester products, due to the higher polarity of the free carboxylic acid group. This order of elution corresponds to that previously reported for 4hydroxybenzoic acid glucoside and its glucosyl ester [15]. Therefore, the W243 mutants have the ability to transfer glucose to both hydroxyl groups to produce glucoside linkages and carboxylic group to produce ester linkages, while wild type recombinant enzyme can produce only ester linkages in detectable amounts.

Aside greater nucleophilicity of the carboxylate groups, the cinnamic acid derivatives might form aromatic stacking interactions with the W243 indole, while their carboxylate reaches in to displace the enzyme from the glucose, thereby favoring glycosylation of the carboxylate in the wild type enzyme. However, the shorter substrates, such as 4HBA and vanillic acid, are likely to stack upon F363, which is in the position of a conserved tryptophan found to stack aglycones in plant GH1 $\beta$-glucosidases with solved crystal structures (e.g. W378 Zea mays Glu1) [34]. Therefore, the main effect of W243 may be in shaping the active site environment and providing a relatively hydrophobic entrance to the site.

The W243 mutations had differential effects on glycosylation of various acceptors (Figs. 2E-J). Higher rates for hydrolysis relative to the best acceptor tested were seen for W243D (69\% of the rate transfer to NAA), W243Y (59\% the rate of transfer to naphthol and trans-cinnaminic acid), W243F (39\% the rate of transfer to 4HBA), and W243N (39\% the rate of transfer to 4hydroxy-benzoic acid). It should be noted that this partly reflects the preference of the various mutants for the phenolic compounds tested. Since D243 and N243 are small and polar relative to W243, it might be expected they would help provide a more hydrophilic active site that could favor water as an acceptor. The Y243 residue might also provide hydrogen bonding to a water network that could help position a water in a position from hydrolysis, but it is the fact that the W243F mutation, in which $\mathrm{W}$ is replaced with the smaller but more hydrophobic F, also increased hydrolysis relative to transglycosylation suggests that the size and interactions with nearby residues may also have effects.

\subsection{Determination of the glucosylation position and fragmentation patterns of kaempferol $O$ -} glucosides 
To further explore the regiospecificity of the Os9BGlu31 W243N mutant in glycosylation of hydroxyl groups compared to the recombinant Os9BGlu31 wild type enzyme, we analyzed the glucosylation positions on kaempferol, a flavonol with four hydroxyl groups to which glucose can be attached, which thus provides a good model system to study regiospecificity. The W243N mutant transferred glucose to the kaempferol acceptor at multiple positions to provide 3 monoglucosides and 3 diglucosides (peaks or compounds 4-6 and 1-3, respectively, in Fig. 4), while wild type Os9BGlu31 produced only one kaempferol monoglucoside under the same conditions, as shown in the chromatograms in Figure 4. Injection of a kaempferol 3-O- $\beta$-Dglucoside standard showed that it co-eluted with 4NP, and the extracted single ion mass chromatogram showed that it is present as a product in the reaction with the Os9BGlu31 W243N, but not the wild type (data not shown).

The glycosylation position has a significant influence on the fragmentation behavior of kaempferol-O-glucosides (Table 1). The fragmentation behavior of kaempferol glucosides and the relative abundance of their radical aglycone ions correlate with glucosylation position. The probable fragmentation pattern of kaempferol 3-O-, 7-O-, and 4'-O-glucosylated isomers of kaempferol are shown in Scheme 1 and supported by the mass spectra in Fig. 5 D-F. These fragmentation patterns are expected from the resonance structures for the radicals created by homolytic cleavage, as illustrated in Scheme 2. As shown, homolytic cleavage of the 3-O-and 4'-O-glucosides will result in radical delocalization over the whole structure, thereby stabilizing these structures and resulting in a greater abundance of the immediate products shown in Scheme 1. In contrast, the 5-O- and 7-O-glucosides do not have as extensive delocalization, so homolytic cleavage is less favored. This explains the differences observed in the mass spectra of 3-O-, 7-O, and 4'-O-glucosylated isomers of kaempferol, specifically in relation to the relative abundances of aglycone $\left[\mathrm{Y}_{0}\right]^{-}$and radical aglycone $\left[\mathrm{Y}_{0}-\mathrm{H}\right]^{-1}$ ions. Compound 4 was annotated as kaempferol 3- $O$-glucoside based on its retention time and fragmentation pattern by comparison with kaempferol 3-O-glucoside standard. Compound 6 showed a similar homolytic cleavage and was thus annotated as kaempferol 4'-O-glucoside. Although the fragmentation pattern of compound 5 is consistent with either kaempferol 5-O-glucoside or kaempferol 7-O-glucoside, the kaempferol 7-O-glucoside standard eluted at the same position and gave essentially the same fragmentation pattern (data not shown), so compound 5 was tentatively annotated at kaempferol 7-O-glucoside. 
The fragmentation behavior of these isomers displayed the predicted ion $\left[\mathrm{Y}_{0}-\mathrm{CO}-2 \mathrm{H}\right]^{-}$, of $m / z$ 255, for compound 4 (Fig. 5D) and the ion $\left[\mathrm{Y}_{0}-\mathrm{CO}\right]^{-}$, of $m / z 257$ for compound 5 (Fig. 5E) [24]; on the other hand, corresponding ions were not observed in the spectrum of compound 6, which is glycosylated on the other aromatic ring, suggesting that the $\left[\mathrm{Y}_{0}-\mathrm{H}\right]^{-1}$ ion at $m / z, 284$ is the precursor of $\left[\mathrm{Y}_{0} \mathrm{H} \mathrm{CO}-\mathrm{H}\right]^{-}$, while the $\mathrm{Y}_{0}^{-}$ion at $\mathrm{m} / z 285$ is the precursor of $\left[\mathrm{Y}_{0}-\mathrm{CO}\right]^{-}$. As shown in Fig. 5F, the spectrum of compound 6 lacks the $\left[\mathrm{Y}_{0}-\mathrm{CO}-2 \mathrm{H}\right]^{-}$ion at $m / z 255$, presumably due to the different resonance localization shown in Scheme 2, and this also provides structural information for isomeric differentiation. Based on the observed fragmentation patterns for the mono-glucosides and the principles of stabilization shown in Scheme 2, we were able to similarly annotate compound 1 as kaempferol 3,7-bis- $O$-glucoside, compound 2 as kaempferol 4',7-bis- $O$-glucoside, and compound 3 as kaempferol 3,4'-bis- $O$-glucoside, as explained in Appendix A Section S1 and Schemes S2 and S3.

\subsection{Kaempferol3-O-glucoside as acceptor and donor for Os9BGlu31 W243 mutants}

The provisional identification of kaempferol glycosides allowed us to compare the different W243 mutants for kaempferol glucoside production. Fig. 6 shows that the W243N mutant can transfer glucose from kaempferol 3-O- $\beta$-D-glucoside to the 7-, and 4'- positions, while the wild type recombinant enzyme cannot effectively do so, although a small amount of kaempferol 3,7-bis- $O$-glucoside was seen after an extended $(3 \mathrm{~h})$ reaction, as shown in Figure 7. The W243N mutant can transfer the glucosyl moiety from kaempferol 3-O- $\beta$-D-glucoside to the 7- and 4'- hydroxyl positions of kaempferol aglycone to produce diglucosides (bis- $O$-glucosides) and monoglucosides, as shown in the chromatogram. Thus, mutation of the W243 to a smaller, more polar residue $(\mathrm{N})$ relaxed the donor substrate specificity of Os9BGlu31.

Furthermore, all of the W243 mutant enzymes can also produce kaempferol-bis- $O$ glucosides from kaempferol 3-O-glucoside and kaempferol mono- and bis- $O$-glucosides from kaempferol with 4NPGlc as glucosyl donor, as shown in Figure 7. A time course showed that each product compound continued to build up from $10 \mathrm{~min}$ to $3 \mathrm{~h}$ for the reaction of $4 \mathrm{NPGlc}$ and kaempferol, despite the fact that some mono- $O$-glucosides were converted further to bis- $O$ - 
glucosides (Appendix A Figs. S3 and S4). Since the different variants fall in the same order, in terms of the amount of each product they produced at each time point, this suggests these results reflect the true glycosylation preferences of these enzymes. Production of kaempferol-7-Oglucoside (compound 5) from kaempferol 3-O-glucoside (compound 4) by the W243A mutant (Figure 7E) indicates that this mutant has the highest activity to deglycosylate kaempferol 3glucoside and prefers to transfer to the 7-O-position, whereas the highest activities to transfer to the 4'- $O$ position were from the W243M and W243N mutants (Figure 7F). Kaempferol bis- $O$ glucosides were produced from kaempferol 3-O- $\beta$-D-glucoside (compound 4 ), with the highest amount of kaempferol 3,7-bis-O-glucoside (compound 1) in the reaction with the W243F mutant (Figure 7A), which along with W243Y showed the highest production of kaempferol 7-O- $\beta$-Dglucoside by transglucosylation from 4NPGlc. Kaempferol 4',7-bis-O-glucoside (compound 2) was only produced from the 3-O-glucoside by $\mathrm{W} 243 \mathrm{~N}$, while $\mathrm{W} 243 \mathrm{M}$ could produce this bisglucoside in similar amounts to W243N when 4NPGlc was used as the donor (Figure 7B). Moreover, W243N produced the most kaempferol 3,4'-bis- $O$-glucoside of any Os9BGlu31 variant (Figure 7C). Thus, Os9BGlu31 variants with different amino acids at residue 243 show different donor and acceptor position specificities, which could be used for differential production of 6 types of kaempferol glycosides.

In wild type and each variant, the 7-O-position was the most often glucosylated, but the variants with smaller residues at residue 243 were able to glucosylate other positions more effectively. As might be expected, the W243F and W243Y mutants that maintained the aromatic side-chains, but with less bulk, were most similar to wild type recombinant enzyme, while variants with smaller residues at this position had greater abilities to transfer glucose to the 3 and $4^{\prime}$ positions. In fact, the W243A mutant, where the whole indole ring was removed to leave a methyl side-chain, had the highest activity toward the 3-O position as an acceptor or donor. Nonetheless, bulk was not the only factor, since the hydrophilic asparagine and aspartate sidechains and the hydrophobic methionine side-chain gave higher activity than alanine for glycosylation at the 4'-hydroxyl position.

In common with the Os9BGlu31 transglucosidase, the residue corresponding to W243 is an important determinant of substrate specificity in GH1 $\beta$-glucosidases [32-33]. The 
corresponding $\mathrm{N} 245$ in Os3BGlu7 (rice BGlu1) $\beta$-glucosidase is critical for efficient oligosaccharide hydrolysis. Mutation of this residue to $\mathrm{V}$ decreased the $k_{\text {cat }} / K_{m}$ for cellotriose 15 fold [32], while conversion to $\mathrm{M}$ decreased $k_{c a t} / K_{m} 18$ fold and conversion of the corresponding $\mathrm{H} 252$ to $\mathrm{M}$ in Os4BGlu12 caused a 2.5-fold drop in this value [33]. In contrast, changing Os3BGlu6 M251 to $\mathrm{N}$ resulted in a 24 -fold increase in the $k_{\text {cat }} / K_{m}$ for cellotriose and smaller increases for other gluco-oligosaccharides. Here, we have verified that this position is also important for acceptor specificity in transglycosylation by Os9BGlu31 transglucosidase, in which W243 appears to restrict acceptor and donor aglycone usage and the regio-specificity of glucosyl transfer.

\section{Conclusion}

The vacuolar Os9BGlu31 transglucosidase acts to transfer glucose between a broad range of phenolic acids, flavonols, and their 1-O-acyl- $\beta$-D-glucose esters. Rice Os9BGlu31 W243 mutants have high activity on hydroxyl groups of phenolic compounds, including those of kaempferol, from which they can produce mono- and bis- $O$-glucosides with glucosyl substituents at the 3-, 7- and 4'-hydroxyls. The active site cleft residues surrounding the aglycone and acceptor binding site play an important role in the transglucosidase specificity of Os9BGlu31 to transfer glucose to different hydroxyl groups on kaempferol to produce kaempferol glucosides. These results suggest that increasing the polarity and decreasing the size of amino acid sidechains around the active site could increase transglucosylation of certain hydroxyl groups on flavonoids and other aromatic alcohols.

The identification of Os9BGlu31 W243 as a key residue restricting transglycosylation specificity from this analysis will allow engineering the enzyme for production of products of interest, as well as assessment of the probable substrate specificity in related transglucosidases.

Acknowledgement: We are grateful to Polkit Sangvanich for helpful analysis and discussion. This work was supported by Suranaree University of Technology, the Commission on Higher Education National Research University Project Grant to SUT and the Royal Golden Jubilee 
Ph.D. Program of the Thailand Research Fund grant to JK and JRKC. Further support was provided by a grant from the U.S. Department of Energy to NGL.

Appendix A. Supplementary data 


\section{References}

[1] D. Bowles, E.K. Lim, B. Poppenberger, and F.F. Vaistij. Glycosyltransferases of lipophilic small molecules. Annu. Rev. Plant Biol. 57 (2006) pp. 567-597.

[2] C. Wei, G. Liang, G. Zilong, W. Wensheng, Z. Hongyan, L. Xianqing, Y. Sibin., X. Lizhong, L. Jie. A novel integrated method for large-scale detection, identification and quantification of widely-targeted metabolites: application in study of rice metabolomics. Mol. Plant. 6 (6) (2013) pp. 1769-1780.

[3] L.L. Lairson and S.G. Withers. Mechanistic analogies amongst carbohydrate modifying enzymes. Chem. Comm. (2004) pp. 2243-2248.

[4] J.R. Ketudat Cairns, and A. Esen. B-Glucosidases. Cell Mol. Life Sci. 67 (2010) pp. 33893405.

[5] Y. Matsuba, N. Sasaki, M. Tera, M. Okamura, H. Matsuoka, K. Nakasawa, H. Funabashi, M. Takatsu, M. Saito, H. Matsuoka, K. Nagasawa, and Y. Ozeki. A novel glucosylationreaction on anthocyanins catalyzed by acyl-glucose-dependent glucosyltransferase in the petals of carnation and delphinium. Plant Cell. 22 (2010) pp. 3374-3389.

[6] D. Teze, J. Hendrickx, M. Czjzek, D. Ropartz, Y.H. Sanejouand, V. Tran, C. Tellier, M. Dion. Semi rational approach for converting a GH1 $\beta$-glycosidase into a $\beta$-transglucosidase. PEDS. 27 (1) (2013) pp. 13-19.

[7] L.F. Mackenzie, Q.P. Wang, R.A.J. Warren, and S.G. Withers. Glycosynthases: mutantglycosidases for oligosaccharide synthesis. J. Am. Chem. Soc. 120 (1998) pp. 55835584.

[8] J. Wei, L. Xun, L. Yang, G. Yang, L. Fu, L. Yang, J. Wang, J. Gao, S. Cheng, Q. Duan, C. in, and X. Li. Glycosynthase with broad substrate specificity-an efficient biocatalyst for the construction of oligosaccharide library. Eur. J. Org. Chem. 138 (2013) pp. 2412-2419.

[9] M. Jahn, J. Marles, R.A.J. Warren, and S. G. Withers. Thioglycoligases: mutant glycosidases for thioglycoside synthesis. Angew. Chem. Int. Ed.42 (2003) pp. 532-534. 
[10] J. Kaur, and R. Sharma. Direct evolution an approach to engineer enzymes. Crit. Rev. Biotechnol. 26 (2006) pp. 165-199.

[11] M.J. Baumann, J.M. Eklöf, G. Michel, Åsa. M. Kalla, T.T. Teeri, M. Czjzek, and H. Brumer III. Structural evidence for the evolution of xyloglucanase activity from xyloglucan endotransglycosylases: biological implications for cell wall metabolism. Plant Cell. 19 (2007) pp. 1947-1963.

[12] E.R. Moellering, and C. Benning. Galactoglycerolipid metabolism under stress: a time for remodeling. Trends Plant Sci. 16 (2010) pp. 98-107.

[13] Y. Nishizaki, M. Yasunaga, E. Okamoto, M. Okamoto, Y. Hirose, M. Yamaguchi, Y. Ozeki, and N. Sasaki. p-Hydroxybenzoyl-glucose is a zwitter donor for the biosynthesis of 7-poly acylated anthocyanin in delphinium. Plant Cell. 25 (2013) pp. 4150- 4165.

[14] T. Miyahara, R. Sakiyama, Y. Ozeki, and N. Sasaki. Acyl-glucose-dependent glucosyltransferase catalyzes the final step of anthocyanin formation in Arabidopsis. J. Plant Physiol. 170 (2013) pp. 619-624.

[15] S. Luang, J.I. Cho, B. Mahong, R. Opassiri, T. Akiyama, K. Phasai, J. Komvongsa, N. Sasaki, Y. Hua, T. Matsuba, Y. Ozeki, J.S. Jeon, and J.R. Ketudat Cairns. Rice Os9BGlu31 is a transglucosidase with the capacity to equilibrate phenylpropanoid, flavonoid, and phytohormone glycoconjugates. J. Biol. Chem. 288 (2011) pp. 10111-10123.

[16] R. J. Nijveldt, E. van Nood, D.E.C. van Hoorn, P.G. Boelens, K. van Norren, and P.A.M. van Leeuwen. Flavonoids: a review of probable mechanisms of actions and potential applications. J. Clin. Nutr. 74 (2001) pp. 397-418.

[17] A. Rora, and M.G. Nair M. G. Structure-activity relationships for antioxidant activities of a series of flavonoids in a liposomal system. Free Radical Biol. Med. 24 (1998) pp. 13-55.

[18] J.D. Gaynor, B.R. Buttery, R.I. Buzzell, D.C. Mac Tavish. HPLC separation and relative of kaempferol glycosides in soybean. Chromatographia. 25 (1988) pp. 1049-1053. 
[19] M.N. Irakli, V.F. Samanidou, C.G. Biliaderis, and I.N. Papadoyannis. Simultaneous determination of phenolic acids and flavonoids in rice using solid-phase extraction and RP-HPLC with photodiode array detection. J. Sep. Sci. 35 (2012) pp. 1603-1611.

[20] A. Staszków, B. Swarcewicz, J. Banasiak, D. Muth, M. Jasiński, and M. Stobiecki. LC/MS profiling of flavonoid glycoconjugates isolated from hairy roots, suspension root cell cultures and seedling roots of Medicago truncatula. Metabolomics. 7 (2011) pp. 604-613.

[21] E. Hvattum, and D. Ekeberg. Studying of the collision-induced radical cleavage of flavonoid glycosides using negative electrospray ionization tandem quadrupole mass spectrometry. J. Mass Spectrom. 38 (2003) pp. 43-49.

[22] M. Luczkiewicz, D. Glod, and T.A. Bucinski. LC-DAD UV and LC-MS for the analysis of isoflavonoids and flavones from in vitro and in vivo biomass of Genista tinctoria L. Chromatographia. 60 (2004) pp. 179-185.

[23] N. Fabre, I. Rustan, E. de Hoffmann, and J .Quetin-Leclercq. Determination of flavone, flavonol, and flavonone aglycones by negative ion liquid chromatography electrospray ion trap mass spectrometry. J. Am. Soc. Mass Spectrom. 12 (2001) pp. 707-715.

[24] F. Cuyckens, and M. Claeys. Mass spectrometry in the structural analysis of flavonoids. J. Mass Spectrom. 39 (2004) pp. 1-15.

[25] T. Sinseadka, S. Wongpornchai, and M. Rayanakorn. Quantification of flavonoids in black rice by liquid chromatography negative electrospray ionization tandem mass spectrometry. J. Agri. Food Chem. 60 (2010) pp. 11723-11732.

[26] K. Ablajan, Z. Abliz, X.Y. Shang, J.M. He, R.P. Zhang, and J.G. Shi. Structuralcharacterization of flavonol 3,7-di-O-glycosides and determination of the glycosylation position by using negative ion electrospray ionization tandem mass spectrometry. J. Mass Spectrom. 41 (2006) pp. 352-360.

[27] R. Opassiri, J.R. Ketudat Cairns, T. Akiyama, O. Wara-Aswapati, J. Svasti, and A. Esen, Characterization of a rice $\beta$-glucosidase genes highly expressed in flower and germinating shoot. Plant Sci. 165 (2003) pp. 627-638. 
[28] S. Seshadri, T. Akiyama, R. Opassiri, B. Kuaprasert, J.R. Ketudat Cairns. Structural and enzymatic characterization of Os3BGlu6, a rice $\beta$-glucosidase hydrolyzing hydrophobic glycosides and $(1 \rightarrow 3)$ - and ( $1 \rightarrow 2)$-linked disaccharides. Plant Physiol. 151 (2009) pp. 4758.

[29] M.A. Marti-Renom, A. Stuart, A. Fiser, R. Sanchez, F. Melo, A. Sali. Comparative protein structure modeling of genes and genomes. Annu. Rev. Biophys. Biomol. Struct. 29 (2000) pp. 291-325.

[30] W. Chuenchor, S. Pengthaisong, R.C. Robinson, J. Yuvaniyama, W. Oonanant, D.R. Bevan, A. Esen, C.J. Chen, R. Opassiri, J. Svasti,and J.R. Ketudat Cairns. Structural insights into rice BGlul $\beta$-glucosidase oligosaccharide hydrolysis and transglycosylation. J. Mol. Biol. 377 (4) (2008) pp. 1200-1215.

[31] S. Sansenya, J. Maneesan, and J. R. Ketudat Cairns. Exchanging a single amino acid residue generates or weakens a +2 cello-oligosaccharide binding subsite in rice $\beta$-glucosidases. Carbohydr. Res. 351 (2012) pp. 130-133.

[32] D. Teze,, J. Hendrickx, M. Dion, C. Tellier, V. L. Woods, V. Tran, and Y. H. Sanejouand. Conserved water molecules in family 1 glycosidases: a DXMS and molecular dynamics study. Biochemistry 52 (2013) pp. 5900-5910.

[33] S. Pengthaisong and J. R. Ketudat Cairns. Effects of active site cleft residues on oligosaccharide binding, hydrolysis and glycosynthase activities of rice BGlu1 and its mutants. Protein Sci. 23 (2014) pp. 1738-1752.

[34] M. Czjzek, M. Cicek, V. Zamboni, D. R. Bevan, B. Henrissat, and A. Esen. The mechanism of substrate (aglycone) specificity in beta-glucosidases is revealed by crystal structures of mutant maize beta-glucosidase-DIMBOA, -DIMBOAGlc and-dhurrin complexes. 
Fig. 1. Superposition of a homology model of Os9BGlu31 trans-glucosidase on the crystal structure of the Os3BGlu6 $\beta$-glucosidase covalent intermediate with 2 -fluoro- $\alpha$-D-glucoside (PDB ID: 3GNR). Os9BGlu31 is shown with orange carbons, while the Os3BGlu6 structure carbons are cyan. The putative nucleophilic (acceptor) water in the Os3BGlu6 complex is shown as a red sphere and distances between it and the anomeric carbon of 2-fluoroglucose and the catalytic acid base (E178) are indicated. The protein backbones are shown in ribbon form. The side-chains of residues surrounding the proposed water/acceptor/aglycone binding site that were significantly less polar in Os9BGlu31 compared to Os3BGlu6, the catalytic acid base residues, and the 2-fluoroglucose-bound nucleophile of Os3BGlu6 are shown in thick stick representations with labels for Os3BGlu6 on top and Os9BGlu31 on the bottom, while other side-chains are shown as lines. Os9BGlu31 residues I172, L183, and L241 were within $5.5 \AA$ of the acceptor water binding site in this superposition, while W243 (8.7 $\AA$ away) was also chosen for mutagenesis due to the importance of the corresponding residue in the substrate specificities of Os3BGlu6, Os3BGlu7 and Os4BGlu12. 
Fig. 2. Relative rates of recombinant Os9BGlu31 enzyme and its active site cleft mutants with various acceptors. Activities were tested with $5 \mathrm{mM} 4 \mathrm{NPGlc}$ donor and $0.25 \mathrm{mM}$ acceptor substrates. 4NP released in 15 min with the appropriate amount of enzyme to give a linear response vs. time was detected by UPLC (Agilent 1290, USA). A comparison of the relative activity levels for the different variant enzymes with ferulic acid acceptor is shown in A. The relative activities with 11 different acceptors (including water for hydrolysis as the last bar) are shown in for wild type Os9BGlu31 (B), I173T (C), L183Q(D), W243A(E), W243D (F), W243F(G), W243M(H), W243N(I), and W243Y(J).

Fig. 3. Chromatograms of reaction mixtures of transfer of glucose to ferulic acid acceptor by Os9BGlu31 wild type and W243 variants. The ferulic acid glucoside peak elutes at approx. 5.5 min, while the glucose ester elutes at $7.4 \mathrm{~min}$. In addition, the chromatograms contain a cluster of buffer peaks centered around $1 \mathrm{~min}$, a large 4NPGlc substrate peak at approx. 6 min, ferulic acid (FA) peaks at 9.5 and $10.2 \mathrm{~min}$, and a 4-nitrophenol (4NP) peak at $11-11.2 \mathrm{~min}$. The mass spectra to the lower left show that both the glucoside (5.5. min) and glucose ester (7.4 min) give the same $[\mathrm{M}-\mathrm{H}]^{-}$peak at 355.1 , along with a dimer at $\mathrm{m} / \mathrm{z} 711$, but with different fragmentation patterns and abundances. Most variants with mutations at W243 can transfer to both hydroxyl and carboxylic acid groups. Transglucosylation products with other acceptors are shown in Figure S2 (Appendix A).

Fig. 4. Chromatogram of transglycosylation products from kaempferol by Os9BGlu31 wild type and W243N mutant using 4NPGlc as a glucosyl donor. The UPLC-MSMS chromatograms of the deduced products in negative ion mode are shown with the provisionally identified products labeled with numbers as follows: kaempferol 3,7-bis- $O$-glucoside (1); kaempferol 4',7-bis- $O$ glucoside (2); kaempferol 3,4'-bis- $O$-glucoside (3); kaempferol 3-O-glucoside and 4NP (4); kaempferol 7-O-glucoside (5); kaempferol 4'-O-glucoside (6); and kaempferol (7).

Fig. 5. $[\mathrm{M}-\mathrm{H}]^{-}$product ion spectra of deprotonated compounds $1,2,3,4,5$, and 6 in the negative ion mode: (A) kaempferol 3-O-glucoside ( $\mathrm{m} / \mathrm{z} 447)$; (B) kaempferol 7- $O$-glucoside ( $\mathrm{m} / \mathrm{z}$ 447); (C kaempferol 4'-O-glucoside ( $\mathrm{m} / \mathrm{z}$ 447): (D kaempferol 3,7-bis- $O$-glucoside $(\mathrm{m} / \mathrm{z}$ 609); (E) kaempferol 4',7-bis- $O$-glucoside ( $m / z, 609$ kaempferol 3,4'-bis- $O$-glucoside $(\mathrm{m} / \mathrm{z}, 609)$. 
Fig. 6. The LC-MS chromatogram of Os9BGlu31 wild type recombinant enzyme and W243N mutant reaction products with kaempferol 3-O-glucoside alone as substrate. For W243N, products are at retention times of $7.6(\mathrm{~m} / \mathrm{z}, 609), 11.2(\mathrm{~m} / \mathrm{z}, 447)$, and $11.5 \mathrm{~min}$ is $(\mathrm{m} / \mathrm{z} 447)$.

Fig. 7. Comparison of kaempferol transglycosylation reaction products from Os9BGlu31 wild type and W243 mutants. The dark bars indicate the amounts of the indicated product or remaining substrate in reactions with kaempferol-3- $O$-glucoside as the lone substrate after $3 \mathrm{~h}$. The light gray bars indicate the amounts of the indicated product produced in reactions with kaempferol as acceptor and 4NPGlc as the donor substrate after $3 \mathrm{~h}$. The reaction products continued to build up to $3 \mathrm{~h}$, as shown in the time course in Fig. S3 (Appendix A), and similar patterns of relative amounts produced by the different Os9BGlu31 variants were obtained at 10, 60 and 180 min (Fig. S4, Appendix A), suggesting the patterns reflect true differences in regiospecificity between the variants.

Scheme 1. Proposed fragmentation pathways of kaempferol mono- $O$-glucosides and possible pathways for the formation of radical ions based on the MS/MS spectra.

Scheme 2. Radical delocalization positions from homolytic cleavage-derived ions of glucose from positions 3 (triangles), 5 (unfilled squares), 7 (filled squares), and 4' (circles) of kaempferol molecule. Notice: fewer resonance structures are possible for 5 and 7. 


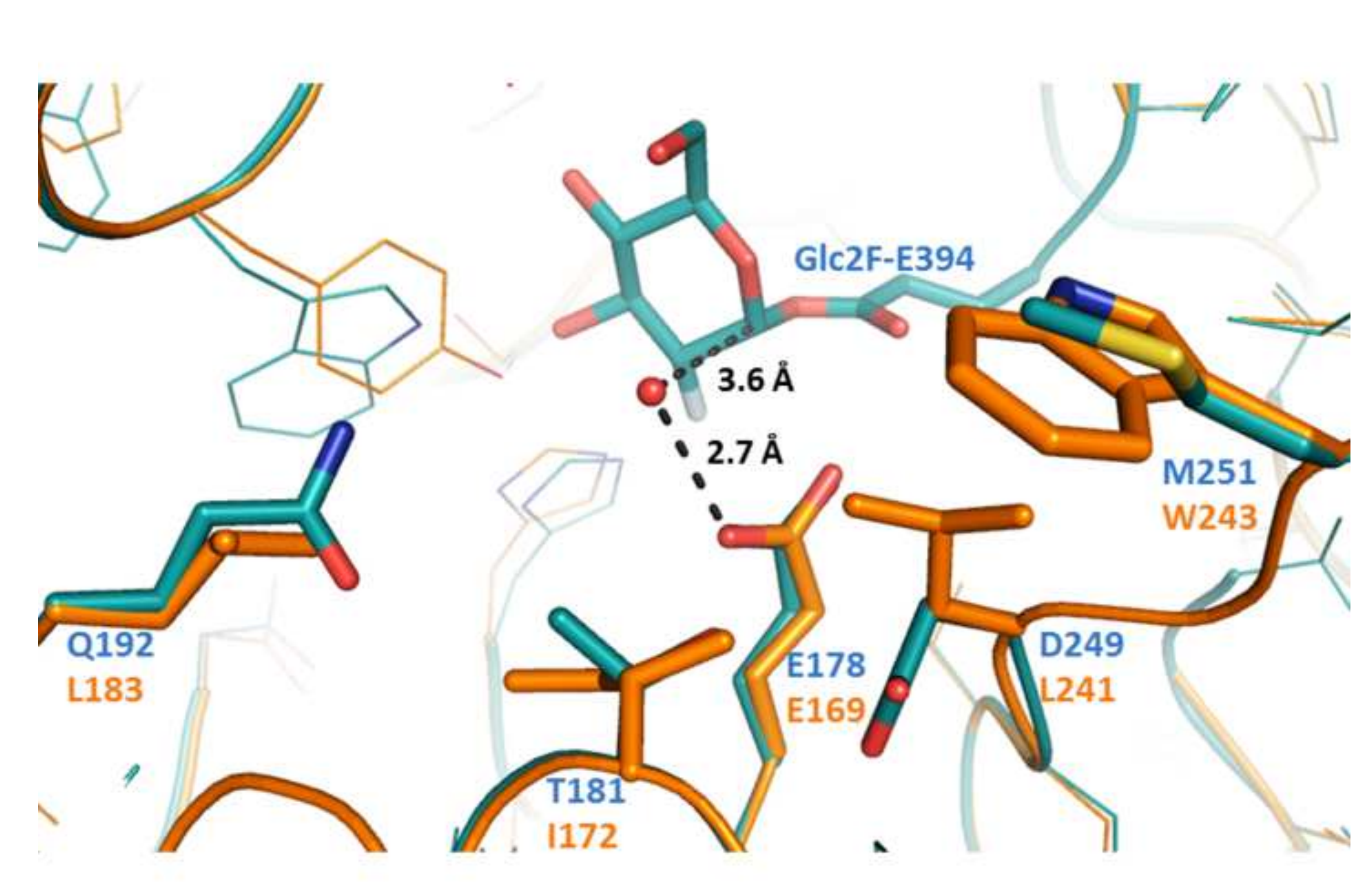

\section{FIGURES}



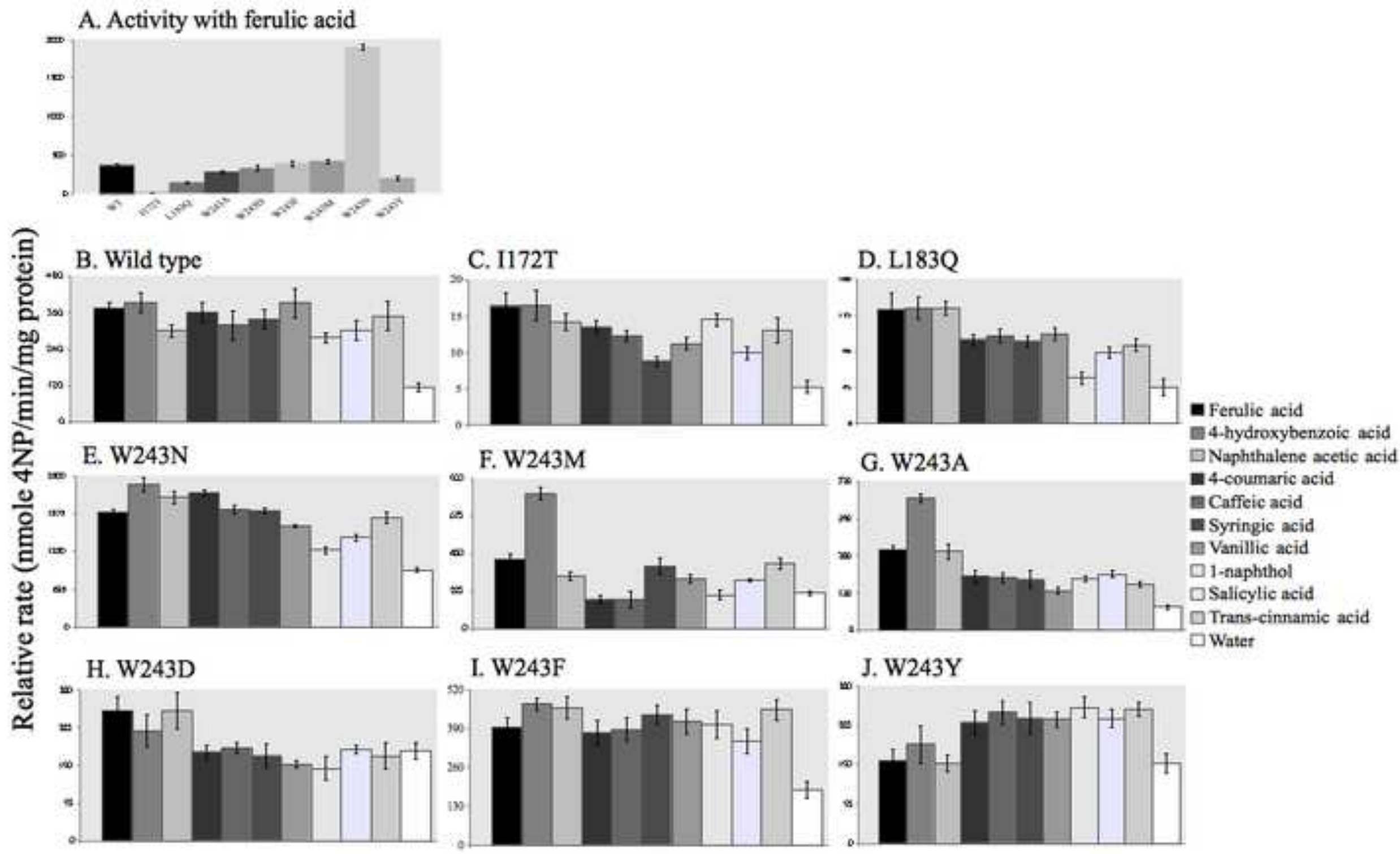


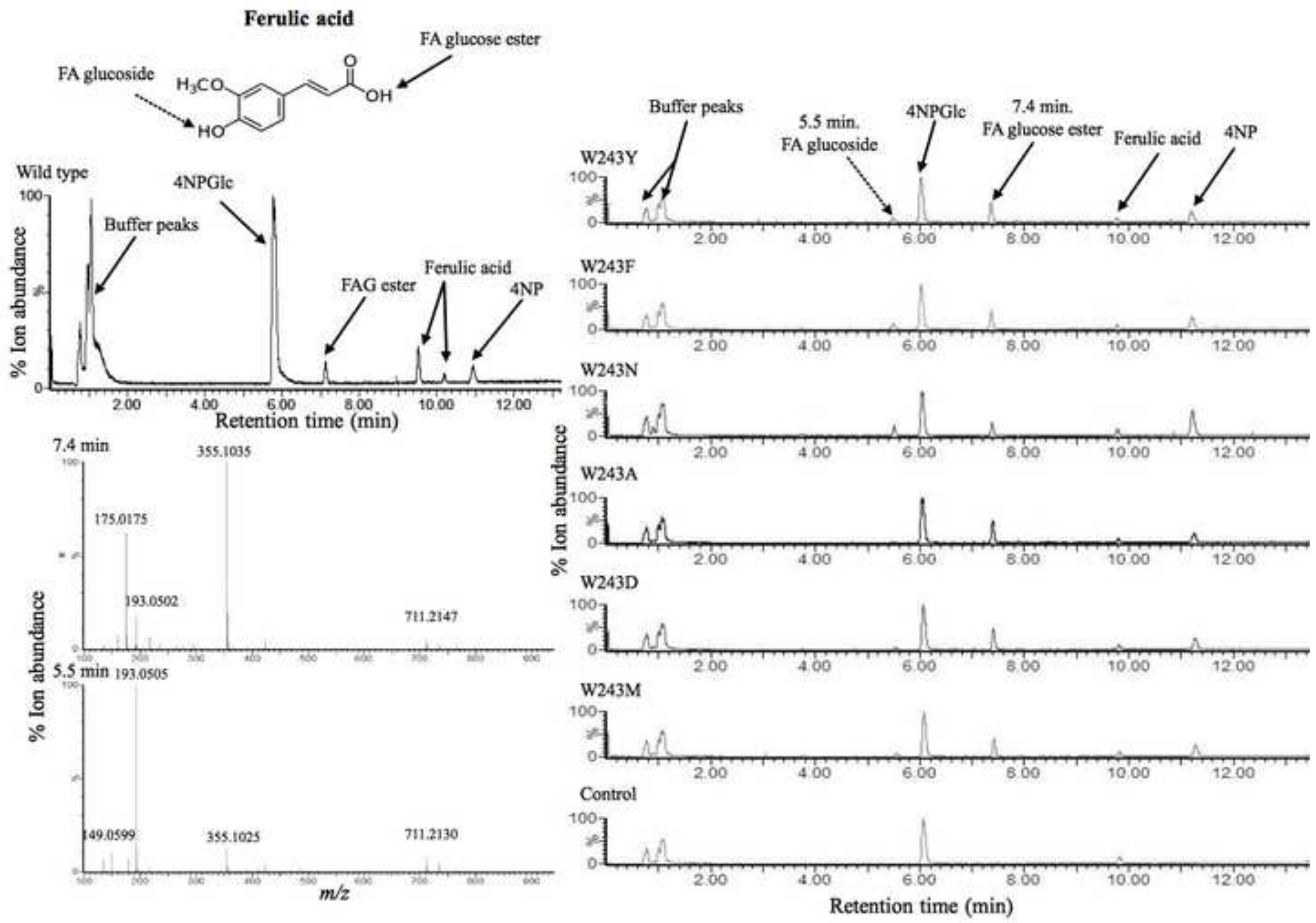


A. Os9BGlu31 W243N mutant

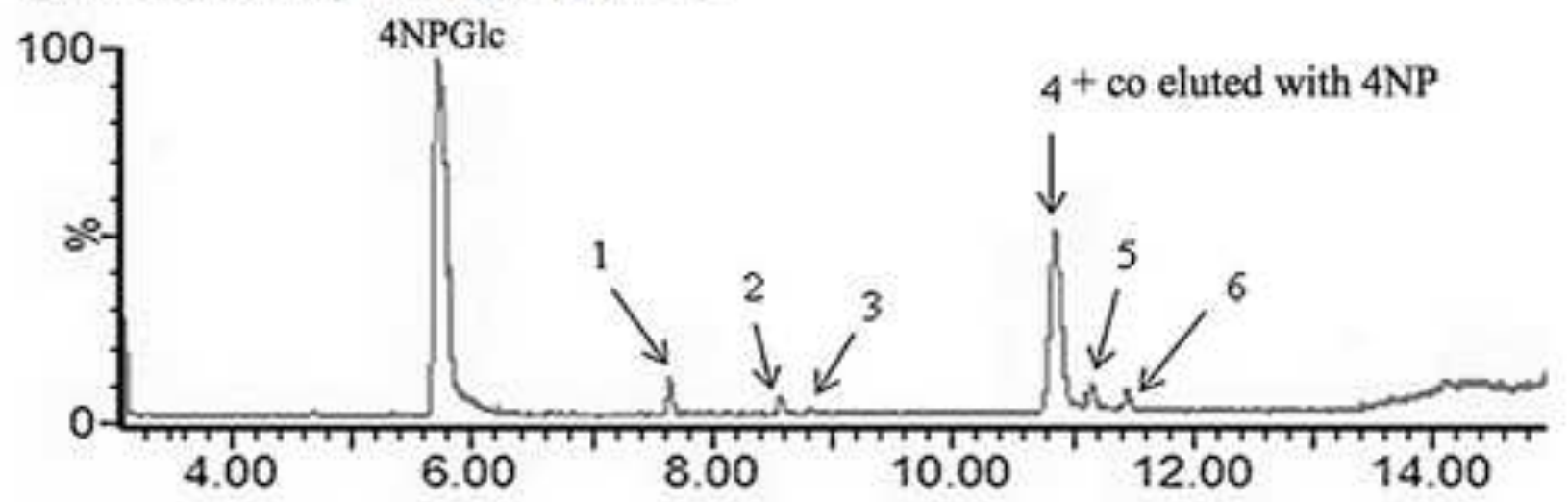

B. Os9BGlu31 wild type

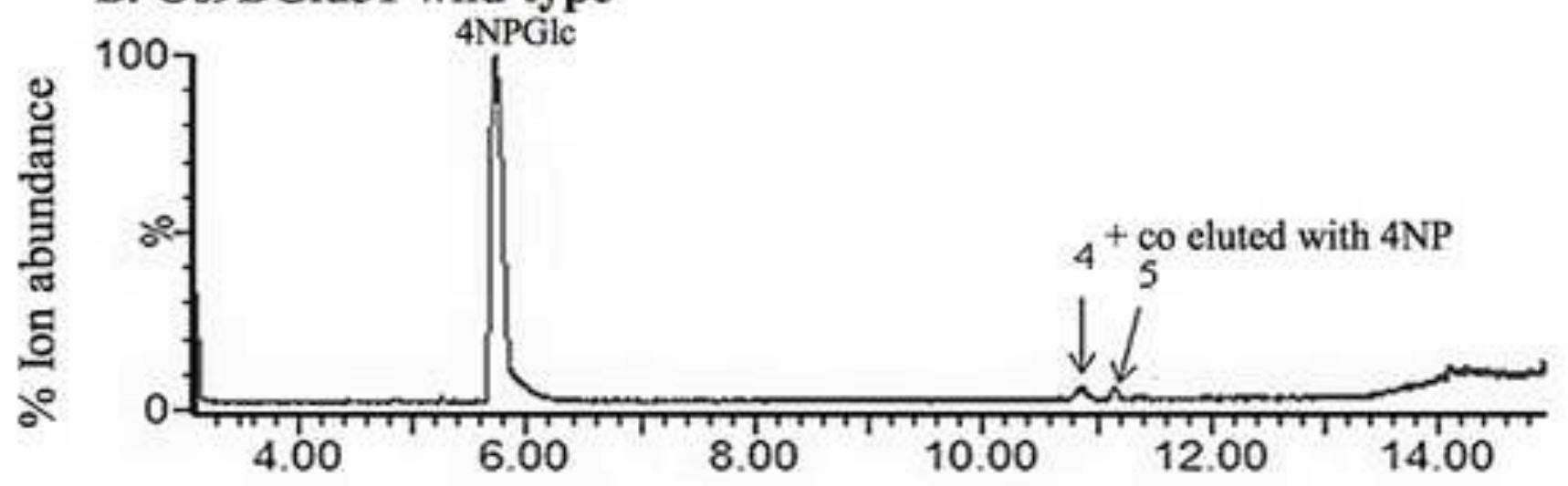

C. Control

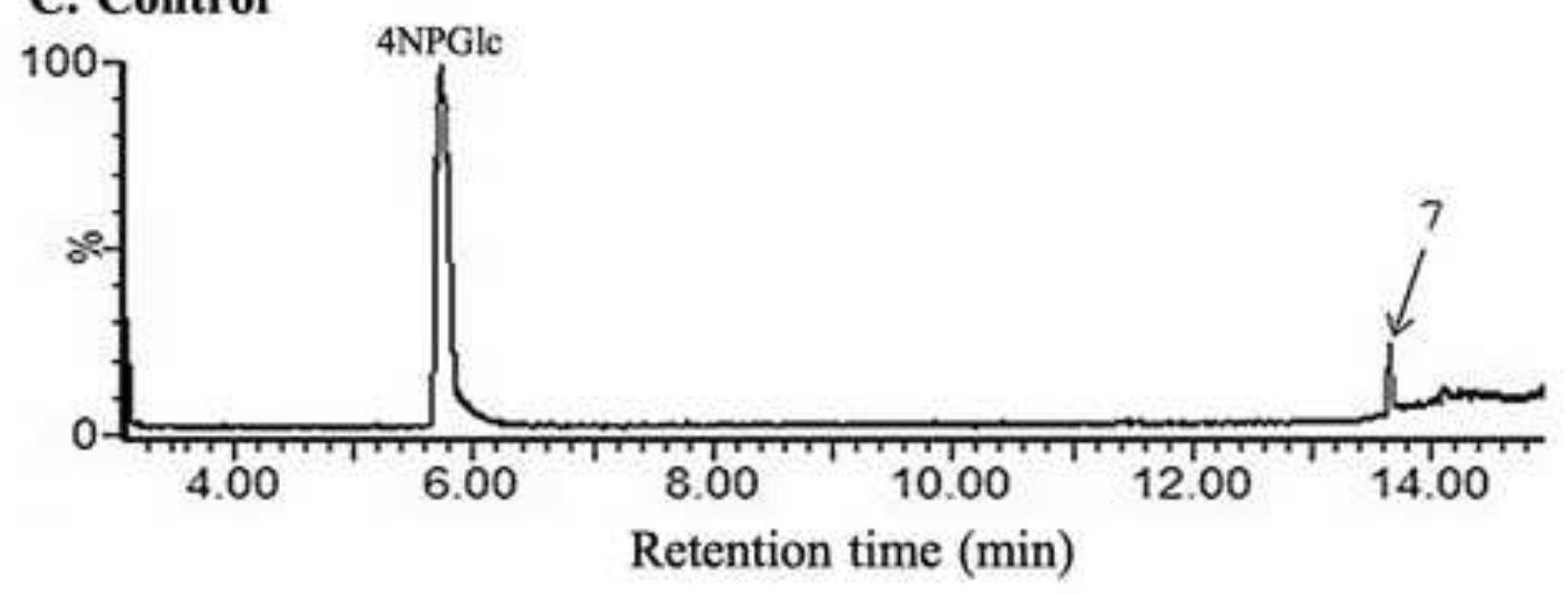


A. kaempferol 3-O-glucoside

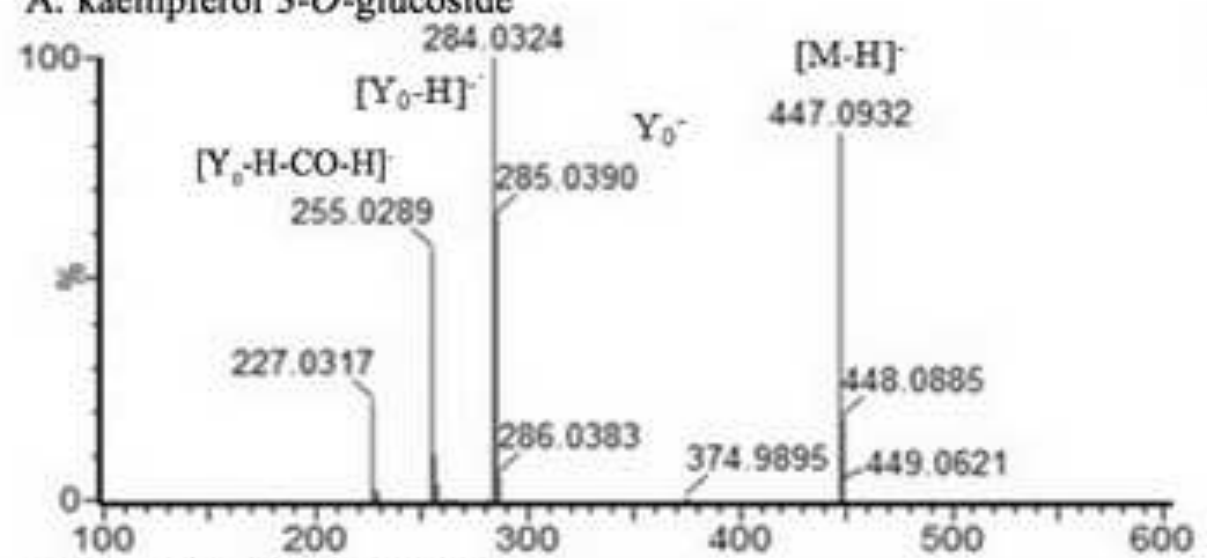

B. kaempferol 7-O-glucoside

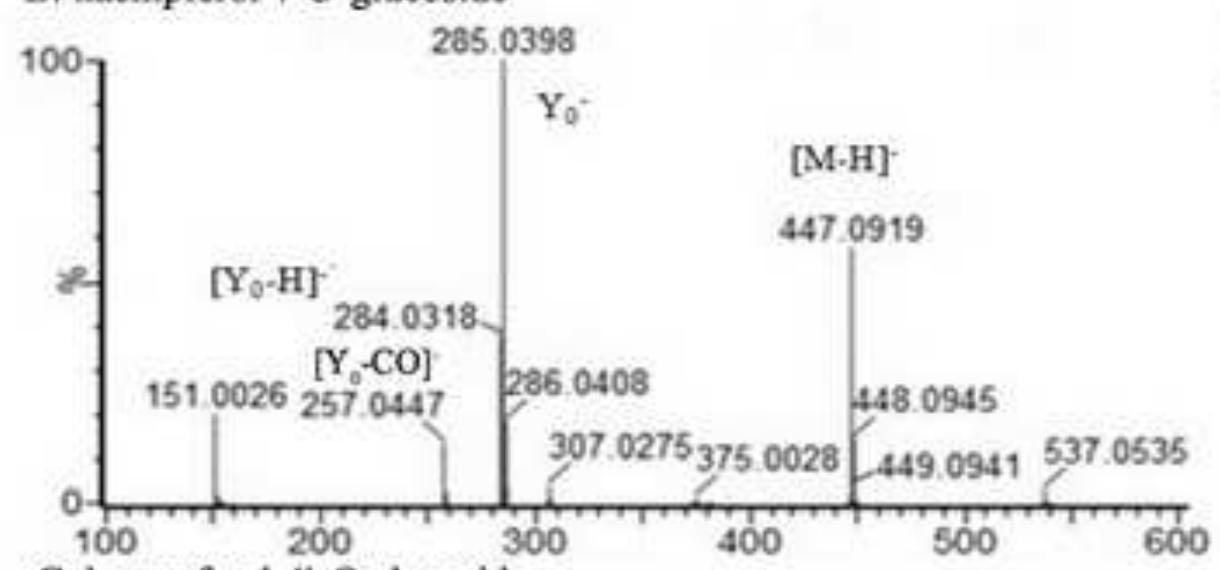

C. kaempferol 4'-O-glucoside

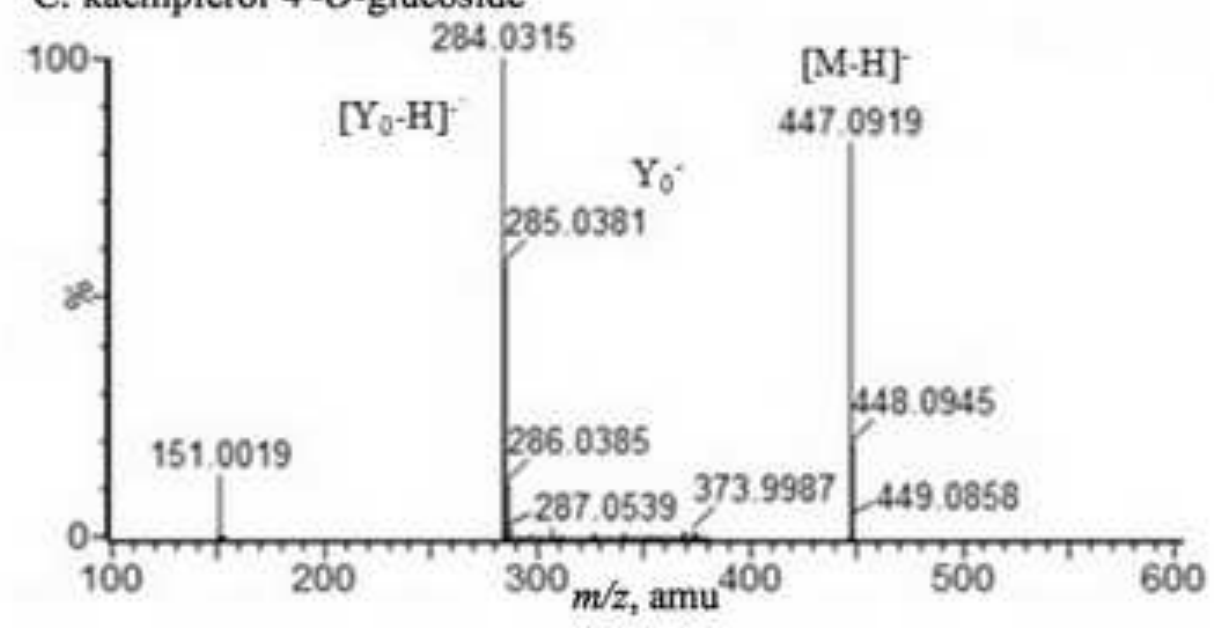

D. kaempferol 3,7-bis-O-glucoside

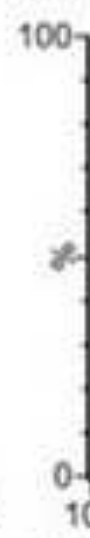

E. kaempferol 4',7-bis-O-glucosic

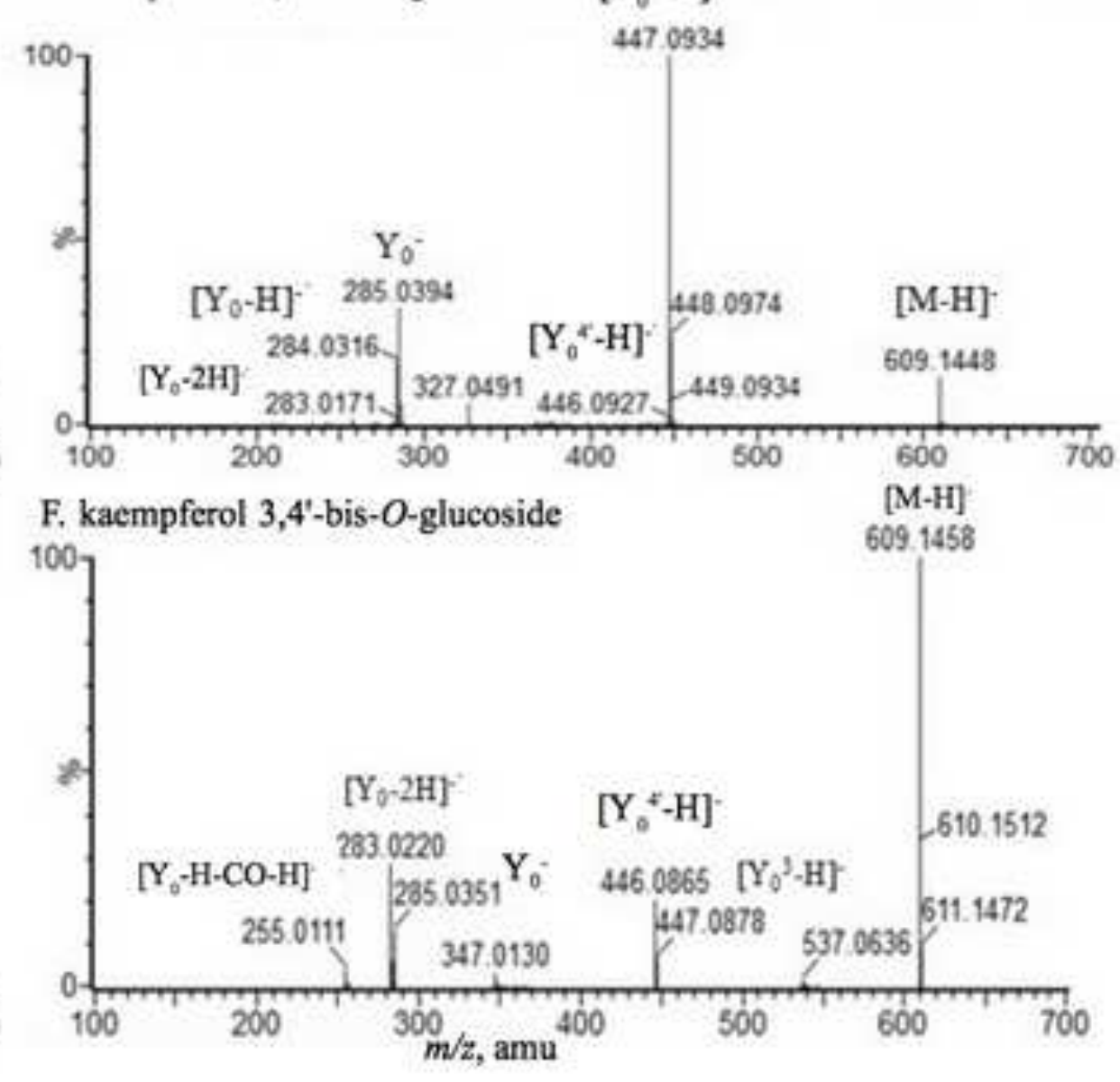

[M-H] 609.1449

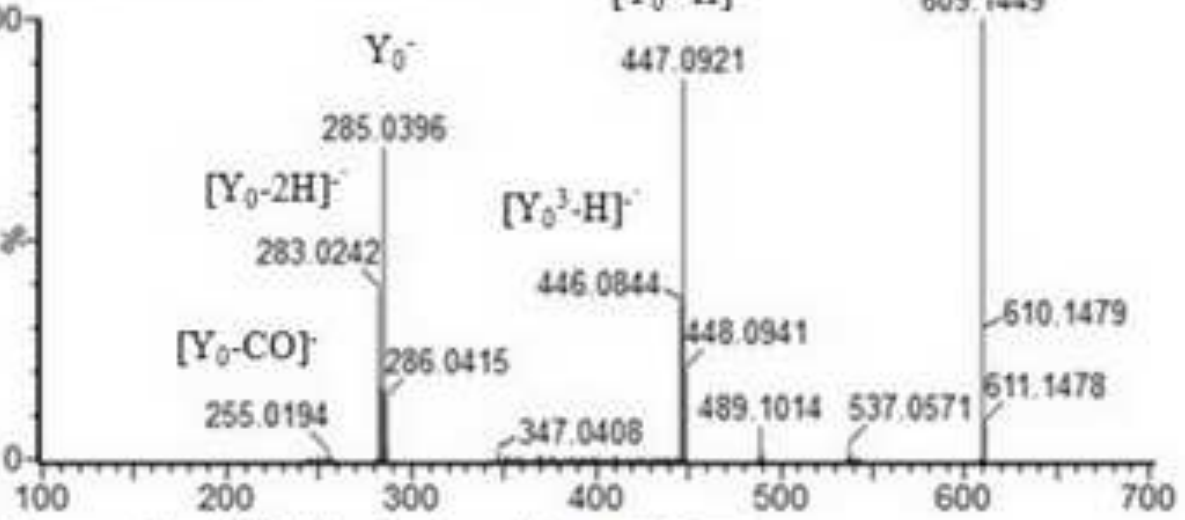
00 
A. Os9BGlu31 W243N mutant

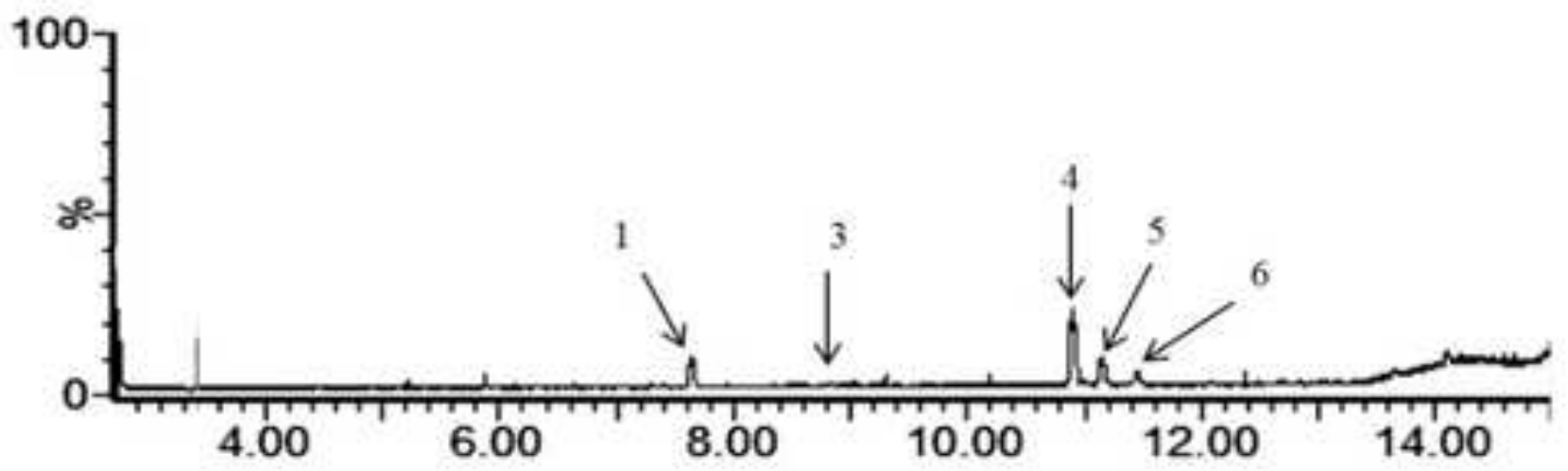

B. Os9BGlu31 wild type

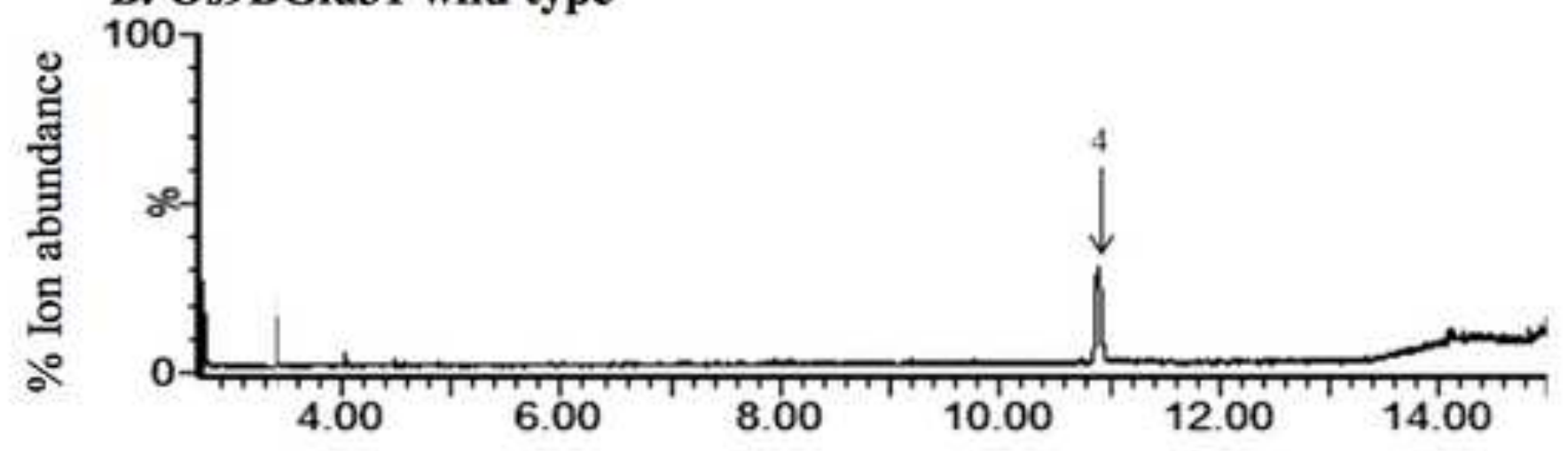

C. Control

100

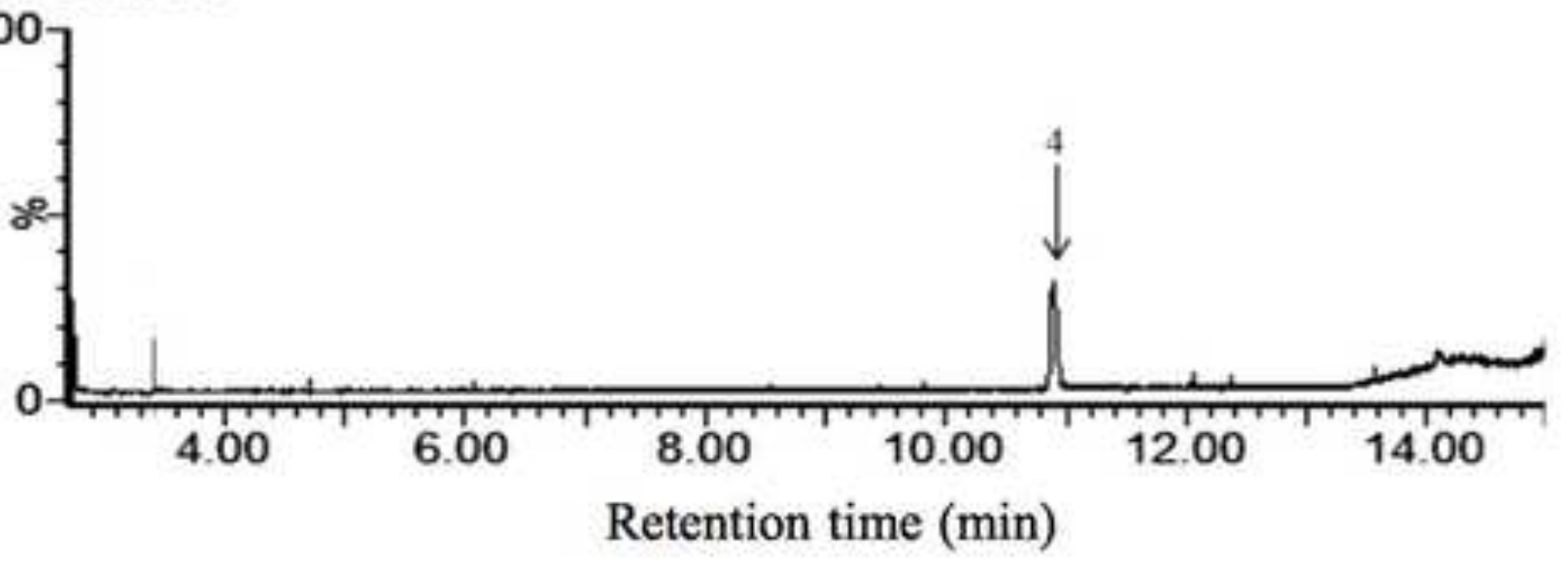



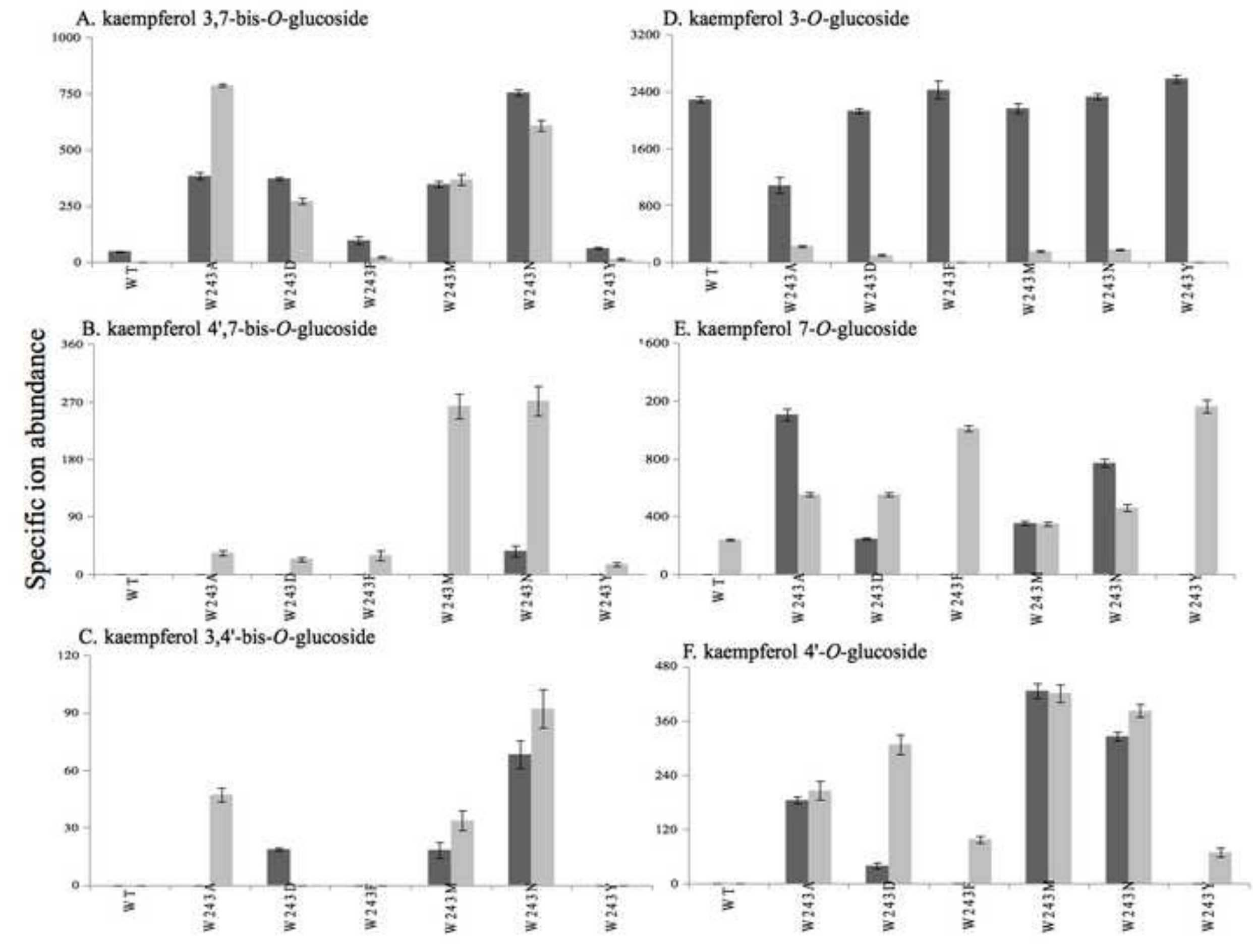

E. kaempferol 7-O-glucoside

C. kaempferol 3,4'-bis-O-glucoside
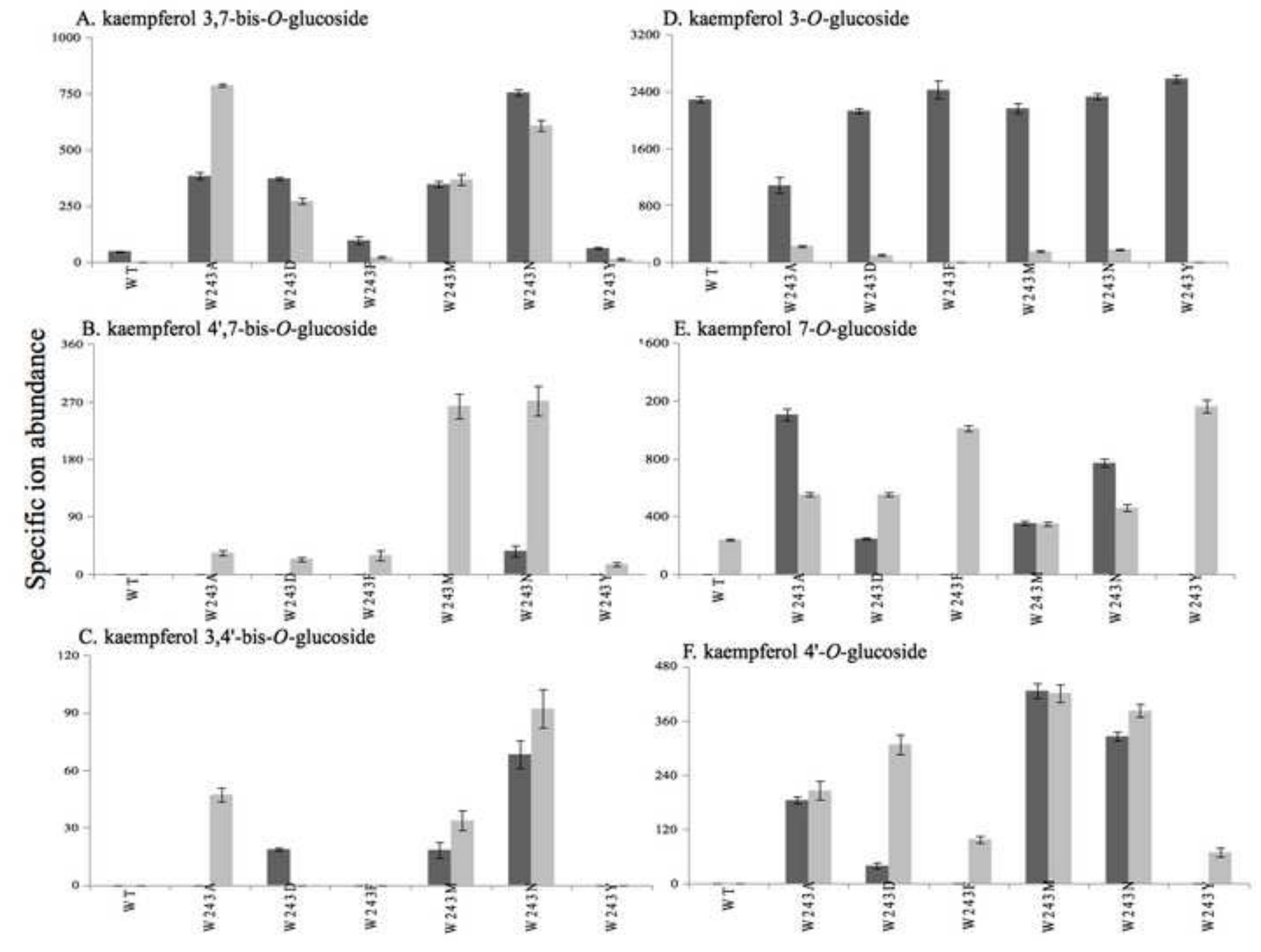
A. kaempferol 3-O-glucoside

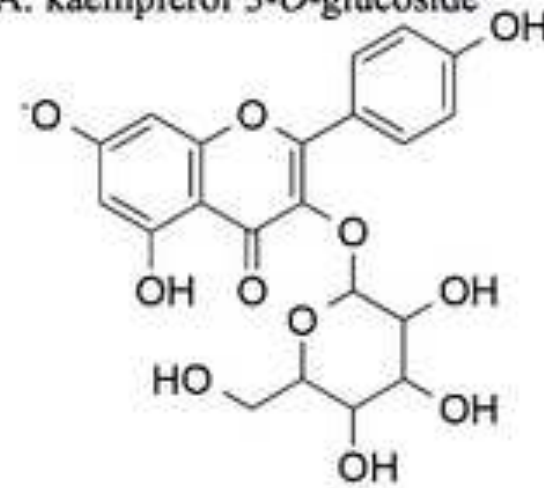<smiles>[Y6][Z10]#[R4]</smiles>

$\stackrel{-\mathrm{CO}-\mathrm{H}^{*}}{ }$

[M-H] $\mathrm{m} / \mathrm{z} 447$

B. kaempferol 7-O-glucoside<smiles>O=c1c(O)c(-c2ccc(Cl)cc2)oc2cc(OC3OC(CO)C(O)C(O)C3O)cc(O)c12</smiles>

[M-H] $\mathrm{m} / \mathrm{z} 447$<smiles>O=c1c(O)c(-c2ccc([O-])cc2)oc2cc(O)cc(O)c12</smiles>

$\mathrm{Y}_{0} \mathrm{~m} / \mathrm{z} 285$

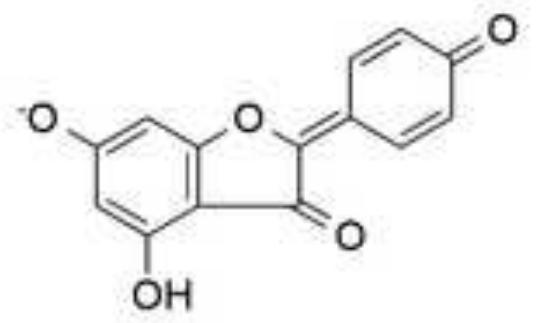

$\left[\mathrm{Y}_{0}-\mathrm{H}-\mathrm{CO}-\mathrm{H}\right] \mathrm{m} / \mathrm{z} 255$

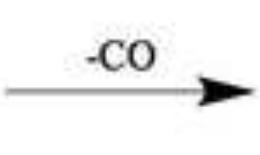

Glc"

[M-H] $\mathrm{m} / \mathrm{z} 447$

$\mathrm{Y}_{0}+\mathrm{m} / \mathrm{z} 284$<smiles>[Y6][R40]#[Y6]</smiles>

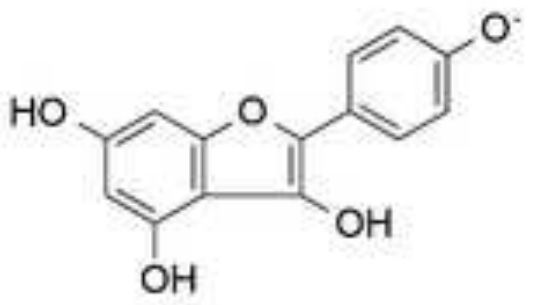

$\left[\mathrm{Y}_{0}-\mathrm{CO}\right] \cdot \mathrm{m} / \mathrm{z} 257$

C. kaempferol 4'-O-glucoside

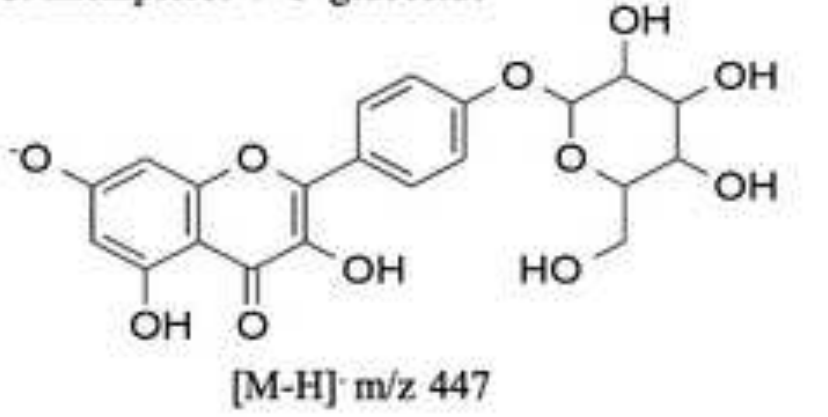



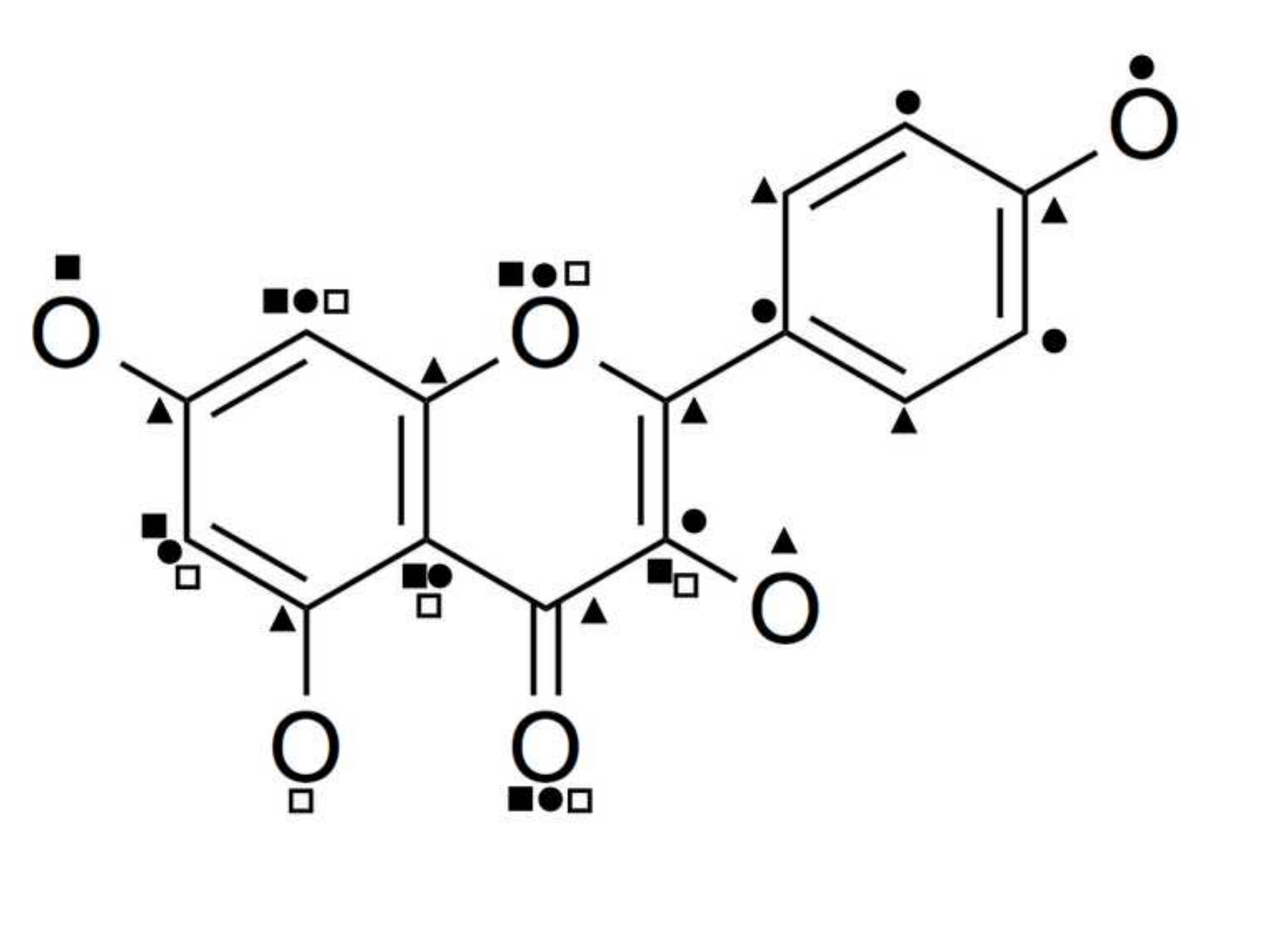